\title{
Performance of a quarter-wavelength particle concentrator
}

\author{
R.J. Townsend ${ }^{\mathrm{a}, *}$, M. Hill ${ }^{\mathrm{a}}$, N.R. Harris ${ }^{\mathrm{b}}$, M.B. McDonnell ${ }^{\mathrm{c}}$ \\ ${ }^{a}$ School of Engineering Sciences, University of Southampton, SO17 1BJ, UK \\ ${ }^{\mathrm{b}}$ School of Electronics and Computer Science, University of Southampton, SO17 1BJ, UK \\ ${ }^{\mathrm{c}}$ Dstl Porton Down, Salisbury, Wiltshire SP4 OJQ UK
}

\section{A R T I C L E I N F O}

Article history:

Received 8 June 2007

Received in revised form 3 June 2008

Accepted 3 June 2008

Available online 14 June 2008

\section{PACS:}

43.25

Keywords:

Acoustic radiation force

Concentration

Particle separation

Suspension

Bio-sensing

\begin{abstract}
A B S T R A C T
A series of devices have been investigated which use acoustic radiation forces to concentrate micron sized particles. These multi-layered resonators use a quarter-wavelength resonance in order to position an acoustic pressure node close to the top surface of a fluid layer such that particles migrate towards this surface. As flow-through devices, it is then possible to collect a concentrate of particulates by drawing off the particle stream and separating it from the clarified fluid and so can operate continuously as opposed to batch processes such as centrifugation.

The methods of construction are described which include a micro-fabricated, wet-etched device and a modular device fabricated using a micro-mill. These use silicon and macor, a machinable glass ceramic, as a carrier layer between the transducer and fluid channel, respectively. Simulations using an acoustic impedance transfer model are used to determine the influence of various design parameters on the acoustic energy density within the fluid layer and the nodal position. Concentration tests have shown up to 4.4-, 6.0- and 3.2-fold increases in concentration for 9, 3 and $1 \mu \mathrm{m}$ diameter polystyrene particles, respectively. The effect of voltage and fluid flow rates on concentration performance is investigated and helps demonstrate the various factors which determine the increase in concentration possible.
\end{abstract}

(c) 2008 Elsevier B.V. All rights reserved.

\section{Introduction}

Multi-layered acoustic resonators for particle manipulation have recently been the subject of much research. These devices rely on the generation of a standing wave in a fluid layer, which causes particles held within this layer to experience acoustic radiation forces $[1,2]$. These particles will typically migrate towards the acoustic pressure node whereby it is possible to form a concentrate. This phenomenon has applications in the handling of biosamples and bio-sensing techniques $[3,4]$ where the sensitivity of a detector depends heavily on the population of events or bio-matter contacting the sensor element or in the detector region. Where particles, cells or spores are of interest, one method to increase the sensitivity of their detection is to concentrate the particles prior to the detection stage.

Here, a device is being developed which is able to continually supply a concentrate of particles by extracting a significant volume of the fluid used to transport the sample. To achieve this, acoustic radiation forces are used to move particles close to a surface where the concentrated particle stream is selectively drawn off. This operation relies on the generation of a quarter-wavelength standing wave which has previously been used to transport particles to a

\footnotetext{
* Corresponding author. Tel.: +44 2380592896; fax: +44 2380597322

E-mail address: R.J.Townsend@soton.ac.uk (R.J. Townsend).
}

surface $[4,5]$ and has paved the way for this study. This previous work has tended to focus on batch processing of a sample (as opposed to continuous flow) and movement of particles up to the surface such that they become adhered to it, ideally where a sensing element is incorporated into the surface. However, the aim here is to process a sample continuously and therefore operate the concentrator as a flow-through device, independent of any sensing stage.

The generation of a near quarter-wavelength acoustic mode is paramount to the successful operation of such a device, but also represents a significant challenge. Currently, a variety of related acoustic devices are being developed by the research community, which rely on a good acoustic design to maximise energy density of the acoustic field and predictable resonant mode. An acoustic impedance transfer model can be used to predict the occurrence of various modes and their strength [6] in a multi-layered device, as is being considered here. When coupled with particle simulations [7], it is a useful tool to assess the likely particle separation.

Acoustic radiation forces typically move solid phase particles to locations of maximum kinetic energy within the acoustic field, i.e. the acoustic pressure node of a standing wave within a simple axial planar resonator. By generating a standing wave in a multi-layered device and controlling the position of the pressure node, it is theoretically possible to move particles to an arbitrary plane within a fluid chamber [8]. These multi-layered devices are typically 


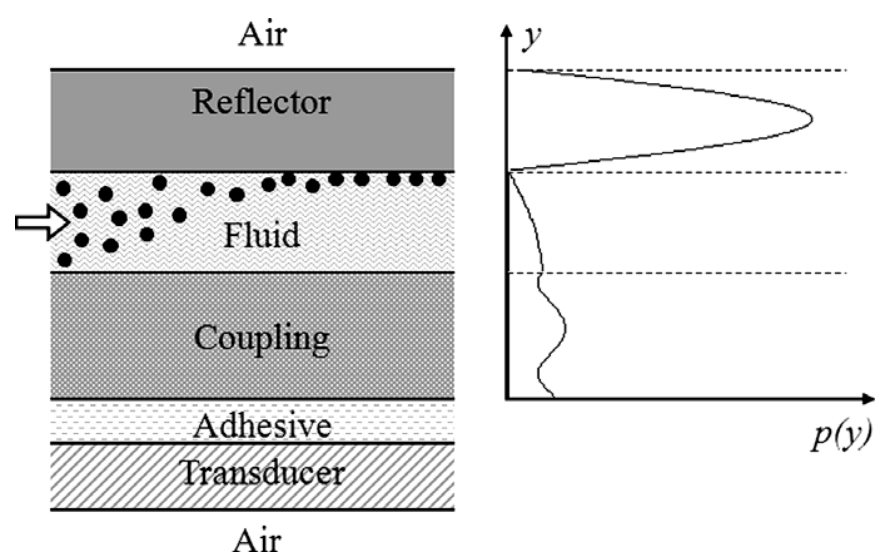

Fig. 1. Schematic of acoustic pressure field within multi-layered resonator.

planar and consist of an ultrasonic piezo-electric transducer, coupling layer, fluid layer and reflector layer with the acoustic field propagating perpendicular to these layers (although ultrasonic particle manipulators are not limited to planar construction [9] or through-thickness plane propagating waves [10]).

Fig. 1 depicts the acoustic pressure field in a multi-layered structure and shows a half-wavelength resonance in the reflector layer which imposes a pressure node close to the reflector surface. Particles suspended in the fluid layer will then migrate to this surface and fluid outlets may be placed across the width of the fluid channel so that certain regions of the fluid can be drawn off. This way a concentrate of particles can be separated from the clarified fluid using two outlet ducts.

As well as a concentrator, a quarter-wavelength device can also operate as filter, removing large particles from smaller particles which experience a lower radiation force. Eq. (1) describes the relationship between the various properties of a particle (radius $R$, density $\rho_{\mathrm{p}}$, and acoustic contrast $\Phi$ ), the wave number $k$, time averaged energy density of the acoustic field $\varepsilon$, and the acoustic radiation force $F_{\mathrm{ac}}[2]$. Eq. (2) defines the time averaged energy density in terms of the real parts of the acoustic velocity field $u(x)$ and pressure field $p(x)$. Finally, Eq. (3) defines the acoustic contrast factor which is a function of the sonic velocities and densities of the particle, $c_{\mathrm{p}}$ and $\rho_{\mathrm{p}}$, and of the fluid, $c_{\mathrm{f}}$ and $\rho_{\mathrm{f}}$, respectively.

$$
\begin{aligned}
& F_{\mathrm{ac}}=4 \pi \varepsilon k R^{3} \Phi(\beta, \rho) \sin (2 k y) \\
& \varepsilon=\frac{1}{2} \rho\left(\mathrm{u}(x)^{2}+\frac{p(x)^{2}}{(\rho c)^{2}}\right) \\
& \Phi=\frac{\rho_{\mathrm{p}}+\frac{2}{3}\left(\rho_{\mathrm{p}}-\rho_{\mathrm{f}}\right)}{2 \rho_{\mathrm{p}}+\rho_{\mathrm{f}}}-\frac{c_{\mathrm{f}}^{2} \rho_{\mathrm{f}}}{3 c_{\mathrm{p}}^{2} \rho_{\mathrm{p}}}
\end{aligned}
$$

It can be seen that $F_{\mathrm{ac}} \propto R^{3}$ therefore the velocity of a particle in a standing wave is highly dependent on the particle size, and thus explains the low forces experienced by smaller particles.

An alternative method of collecting a concentrate has been considered which uses a half-wavelength resonance such that particles migrate to the centre plane of a fluid chamber [11]. More recently, variations of this approach where the acoustic nodal planes are instead generated perpendicular to the transducer have been demonstrated by Nilsson et al. [12] and Petersson et al. [13], with the latter using three outlet channels to separate red blood cells from lipids. In general, a half-wavelength mode is more energetically efficient than a quarter-wavelength as the fluid chamber itself is in resonance. This results in high acoustic radiation forces permitting the use of lower transducer driving voltages or higher flow rates. However, the requirement of three outlets to separate the two regions of clarified fluid and particle stream make it difficult to balance the fluidic system, particularly where the two clarified streams need to be recombined; any mismatch between flow rates through the clarified ducts would disturb the efficient extraction of the particle concentrate.

\section{Wet-etched device}

\subsection{Construction}

The initial studies reported here investigate the formation of a particle concentrate using a micro-fabricated silicon device. A similar device is described by Harris et al. [14] and is pictured schematically in Fig. 2. Anodic bonding is used to assemble wetetched silicon and Pyrex wafers, etched to form a wide (approximately $5 \mathrm{~mm}$ ) yet shallow chamber, which are then diced to form a microfluidic device. Although thick-film printed PZT has been used successfully with these devices, this work relies on bulk PZT plates bonded to the silicon surface using an adhesive.

\subsection{Design}

Simulations were used to predict the position of the node relative to the glass surface for a range of fluid thicknesses, but otherwise the design does not differ significantly from the dual frequency device reported in Harris et al. [15] which exhibited both quarter- and half-wavelength resonances. Simulated results show that a near quarter-wavelength resonance exists for a range of fluid depths. This is illustrated in Fig. 3 where for a resonant system the acoustic pressure field within the fluid cavity has been plotted for a range of fluid cavity thicknesses $t_{\mathrm{f}}$. By selecting this depth the nodal position can be controlled (the fluid cavity is wet-etched into a $1.7 \mathrm{~mm}$ Pyrex wafer therefore, for example, if $t_{\mathrm{f}}=210 \mu \mathrm{m}$, the reflector thickness is $1490 \mu \mathrm{m})$. It can be seen that the acoustic node can be positioned a short distance away from the reflector surface to limit the chance of particles adhering to the surface.

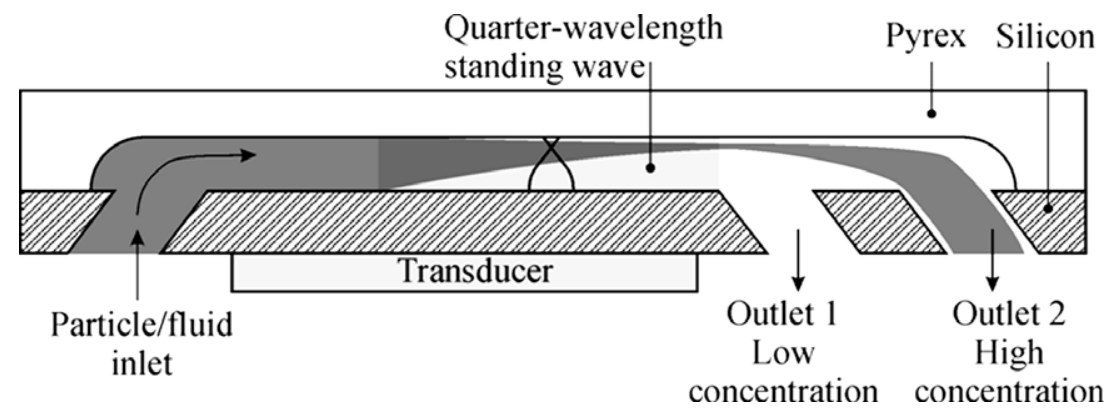

Fig. 2. Schematic of micro-engineered ultrasonic particle separator. 


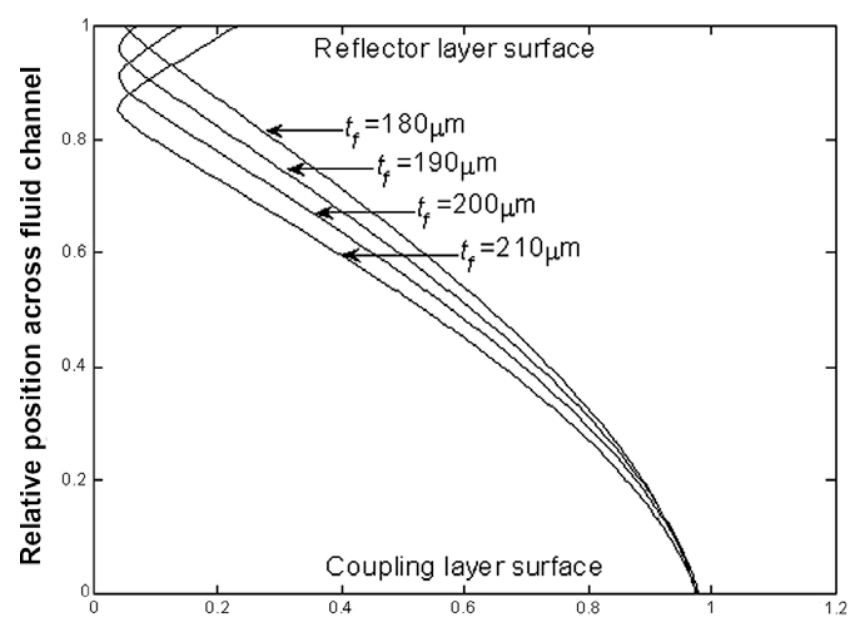

Pressure across fluid (normalised to pressure amplitude)

Fig. 3. Predicted acoustic pressure profile within the fluid layer of the microfabricated concentrator at quarter-wavelength resonant frequency and as a function of position $y / t_{\mathrm{f}}$.

From the figure it is also interesting to note that due to losses in the various layers and attenuation of the reflected wave a zero pressure node is difficult to achieve. A thickness of $200 \mu \mathrm{m}$ was selected for fabrication as listed in Table 1 with the description of the various layers of the resonator structure.

\subsection{Separation results}

During experimental testing, a particle suspension is continually pulled through the device using a dual channel peristaltic pump for which, being a constant displacement pump, the flow rate is dependent on the tubing bore size and speed of the pump. The flow splits quoted in this paper are regularly measured and are limited by the available tubing bore sizes and the relative sizes used in the two channels. Unless otherwise stated, the typical flow rate used during these tests was $\sim 200 \mu \mathrm{l} /$ min corresponding to a mean flow velocity of approximately $3.3 \mathrm{~mm} / \mathrm{s}$. Concentration measurements were made using a haemocytometer.

Fig. $4 \mathrm{a}$ and $\mathrm{b}$ shows how the outlet concentration relative to the inlet varies over a range of transducer voltages and for $9 \mu \mathrm{m}$ and $3 \mu \mathrm{m}$ diameter polystyrene beads respectively. As the voltage is increased, the acoustic energy density and thus the radiation force also increase and this is seen in the slight improvement in concentration through outlet 2. Measurements at the lower voltages indicate that greater separation can be achieved for the larger particles, although for the $9 \mu \mathrm{m}$ particles a fall off in performance is seen at the higher voltages due to trapping of the particles within the main chamber by high lateral radiation forces. Applying a correction factor to the results to account for lost particles, a 6 - or 7-fold increase in concentration can be achieved compared to a maximum possible 8 -fold increase based on the flow split used.

Similar experiments using $1 \mu \mathrm{m}$ diameter particles prove challenging. Although movement of particles towards the pressure node was observed, the measured outlet concentrations did not

Table 1

Specification and nominal properties of micro-engineered device

\begin{tabular}{lcll}
\hline Layer & Thickness $(\mu \mathrm{m})$ & Density $\left(\mathrm{kg} / \mathrm{m}^{3}\right)$ & Sonic velocity $(\mathrm{m} / \mathrm{s})$ \\
\hline Transducer PZ26 & 670 & 7700 & 4080 \\
EpoTek 301 glue & 10 & 1080 & 2640 \\
Silicon & 525 & 2340 & 8430 \\
Fluid cavity & 200 & 1000 & 1500 \\
Pyrex & 1500 & 2240 & 5640 \\
\hline
\end{tabular}
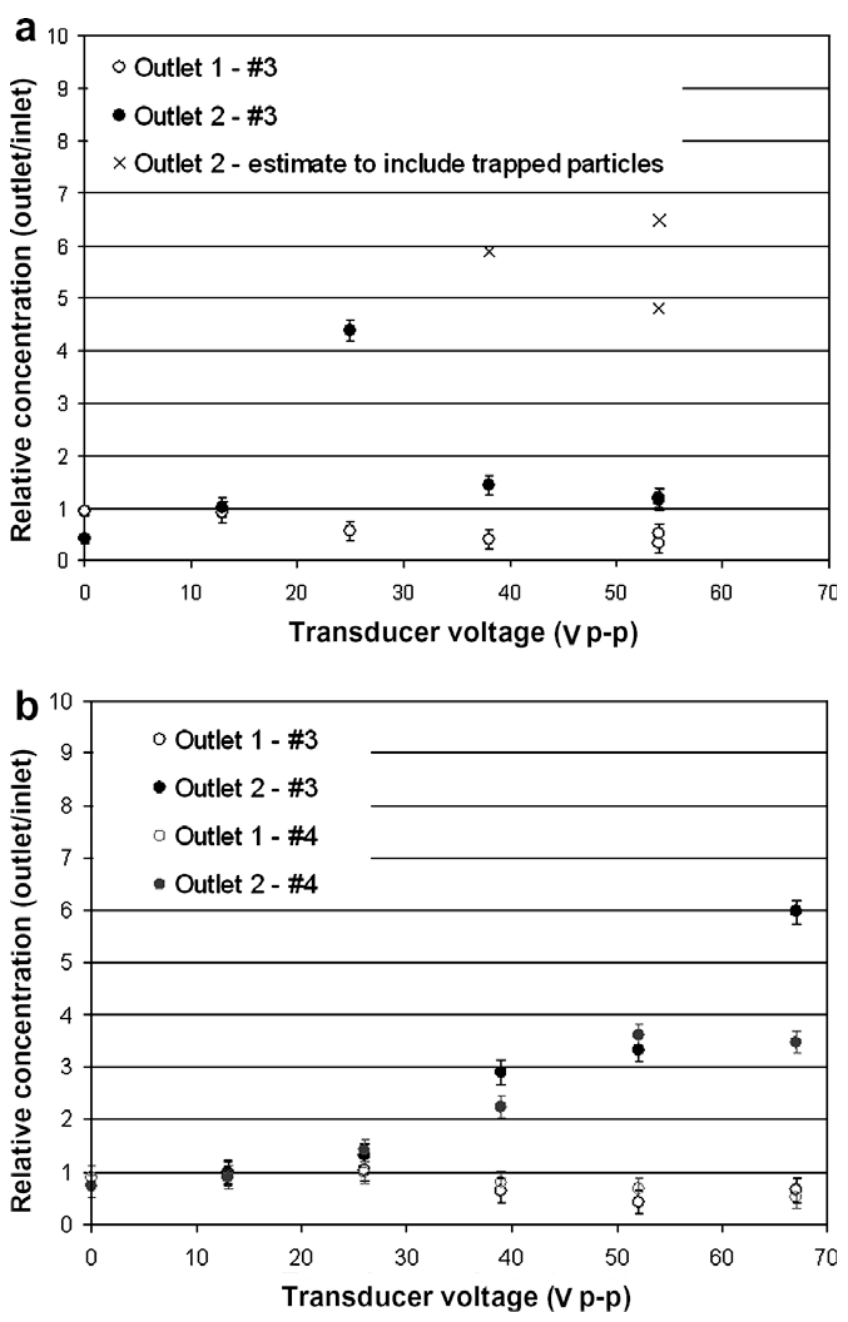

Fig. 4. Measured concentration of (a) $9 \mu \mathrm{m}$ and (b) $3 \mu \mathrm{m}$ diameter polystyrene beads over a range of transducer voltages ( $\left.V_{\text {peak-peak }}\right)$ for an outlet 1 -outlet 2 flow split of 88:12 in the micro-fabricated concentrator. "\#3" and "\#4" refer to the two devices used in this experiment.

conclusively demonstrate the separation process. In the region beyond outlet 1 , the flow velocity becomes significantly lower than that seen in the main channel and trapping of particles by lateral radiation forces against the flow caused particles to be retained between the two outlets. Removal of the field allowed the particles to be carried away with the fluid flow, demonstrating that the trapping was primarily an acoustic effect. In general, it is quite possible to build up a significant concentrate of particles by trapping using acoustic radiation forces, but this is not compatible with continuous processing in a flow-through device.

\section{Modular micro-milled device}

\subsection{Construction}

The wet-etch micro-fabrication approach is suitable for batch runs and tends to be an expensive technique. Therefore, a second phase of this study has used a direct mechanical milling approach which has the advantage of fast turn-around and requires fewer resources than wet-etching. It also lends itself to a more modular approach and allows more flexibility in the acoustic design as compared to the micro-fabricated devices.

A Datron micro-mill has been used to form separate parts of the concentrator and fluid manifold and is able to create small features 
Table 2

Specification and nominal properties of modular, milled device

\begin{tabular}{lrll}
\hline Layer & Thickness $(\mu \mathrm{m})$ & Density $\left(\mathrm{kg} / \mathrm{m}^{3}\right)$ & Sonic velocity $(\mathrm{m} / \mathrm{s})$ \\
\hline Transducer PZ26 & 1000 & 7700 & 4080 \\
EpoTek 301 glue & 10 & 1080 & 2640 \\
Macor & 1200 & 2540 & 5510 \\
Fluid cavity & 180 & 1000 & 1500 \\
Soda lime glass & 1390 & 2240 & 6000 \\
\hline
\end{tabular}

using mill bits down to $0.1 \mathrm{~mm}$ wide and with a depth resolution of $0.01 \mathrm{~mm}$. A glass ceramic, Macor, was chosen to replace the silicon coupling layer due to its good acoustic properties and machinability. Also, soda lime glass microscope slides replaced the Pyrex reflector material as these are more easily available in a variety of thicknesses. A summary of the various layers and their properties can be seen in Table 2 .

During operation, the micro-engineered device and related systems reported in the literature are observed to form striations, which in the case of the micro-engineered device are likely to be a result of acoustic enclosure modes giving rise to lateral acoustic radiation forces [16], although other possible causes include nearfield effects from the transducer, which have been used to trap particles [17], and structural modes in the surrounding material that can be excited deliberately [10]. To suppress enclosure modes and promote a more uniform nodal plane, the compliant silicone gasket material used to seal the main fluid chamber also forms the side-walls of the chamber [16].

This device is shown in Fig. 5 where the fluid chamber can be seen in the centre surrounded by a darker gasket. The assembly is clamped down onto an acrylic manifold which includes three fluidic ports and an electrical connector to the transducer underneath the main chamber.

\subsection{Design}

The acoustic impedance transfer model was again used to select suitable thicknesses for each layer listed in Table 2. There are few limitations to these dimensions due to the variety of glass slides and therefore reflector thicknesses available and the ability to mill other layers to the desired dimensions. This is unlike the microengineered device which is limited by the availability of silicon and Pyrex wafer thicknesses. The results from multiple simulations give a picture of how thickness dimensions of the various layers influence the acoustic energy density within the fluid layer and the position of the node at the resonant frequency. Fig. 6 shows an example of how the coupling layer and reflector thickness influence the energy density in a quarter-wavelength resonant field. The dark bands of the contour plot indicate design solutions in which the energy is particularly low, and interestingly also coincide with $\lambda$ and $3 \lambda / 2$ thicknesses in the transducer and coupling layer. High energy regions exist close to and either side of these bands. Together with results describing the position of the acoustic node, these simulations were used to determine the relative thicknesses of the transducer, coupling, fluid and reflector layers.

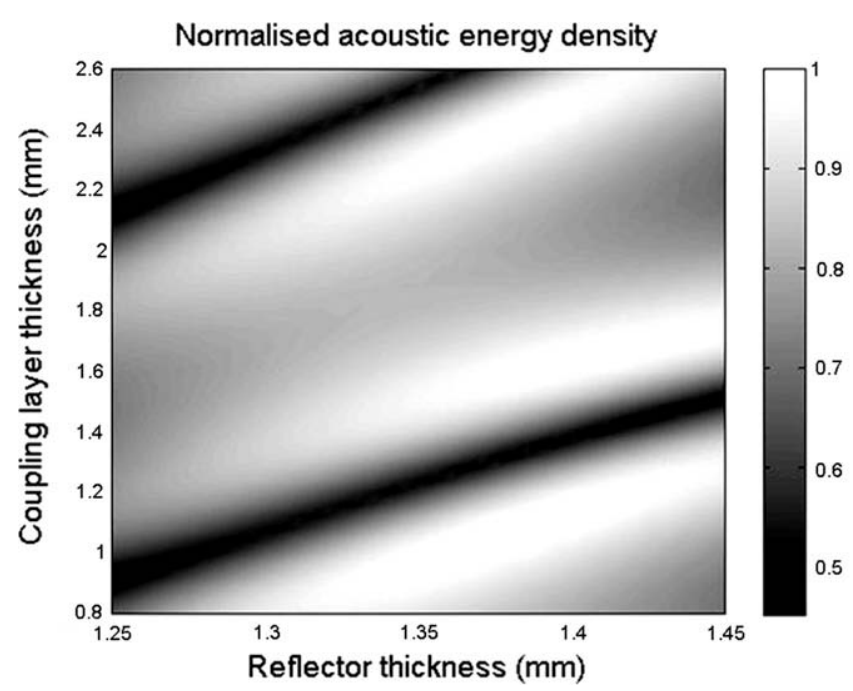

Fig. 6. Example of predicted peak acoustic energy density in fluid layer as a function of Macor coupling layer and reflector thicknesses (where $t_{\mathrm{f}}=180 \mu \mathrm{m}$ ).
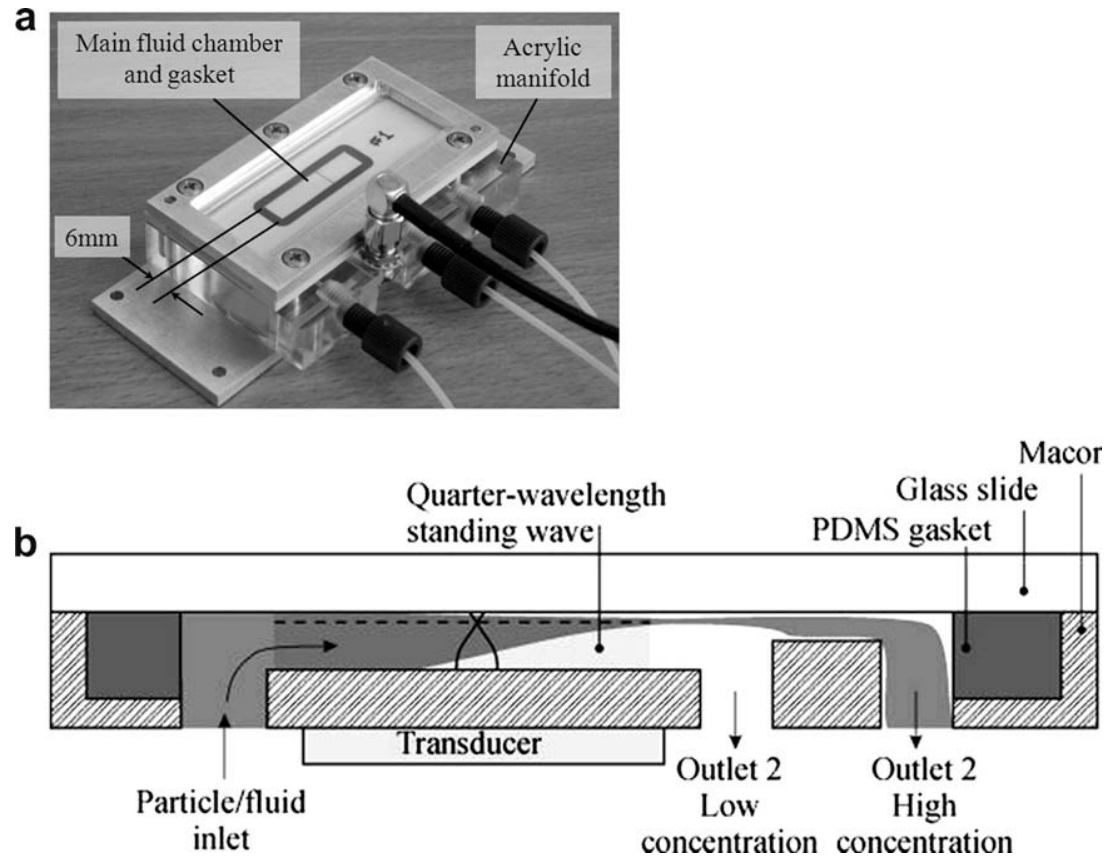

Fig. 5. Photograph (a) and schematic (b) of modular device. 


\subsection{Separation results}

Although previous experiments with the micro-engineered device used an 88:12 flow split to result in a maximum 8-fold increase in concentration, tests with the modular device also included less severe flow splits in an effort to achieve more complete separation of the particles, although with a reduction in the maximum concentration possible.

Table 3 includes some of the separation results collected for 1 and $9 \mu \mathrm{m}$ particles for a flow split of 50:50 and 71:29. In the first set of results shown at a 50:50 flow split, a high proportion of $9 \mu \mathrm{m}$ particles are entrained in the outlet 2 channel with no particles seen in the outlet 1 channel. This contrasts with the $1 \mu \mathrm{m}$ particles which do not appear to be influenced by the acoustic field significantly. It can also be seen that not all particles are accounted for and are likely to be lost within the device itself or adhered to outlet reservoir walls.

The slightly higher proportion of $1 \mu \mathrm{m}$ particles through outlet 1 suggests that gravitational forces are more significant than acoustic radiation forces. This is again demonstrated in the last two sets of data at a 71:29 flow split where orientation is seen to influence the separation of particles. In the normal orientation (reflector layer upper-most) gravitational forces oppose the radiation forces slowing the migration of particles and causing the concentrate to form on a plane slightly beneath the acoustic node. Therefore by inverting the device, particle separation will be aided by gravity, improving the clarity of outlet 1 and concentration of outlet 2. The final set of results represents a 2.9-fold increase in concentration for $9 \mu \mathrm{m}$ particles. However, a maximum of 3.4 would be possible if no particles were lost in the fluid system or during sample handling. This is calculated using Eq. (2) where the concentration increase is divided by the proportion of particles which pass through both outlets 1 and 2 compared to the inlet

Outlet 2 concentration $=D \cdot \frac{A}{B} \cdot \frac{1}{C+D}$

where

$A=$ Inlet flow rate

$B=$ Outlet 2 flow rate

$C=$ Proportion of inlet particles which pass through outlet 1

$D=$ Proportion of inlet particles which pass through outlet 2

To concentrate $1 \mu \mathrm{m}$ diameter particles, it is necessary to either increase the driving voltage of the device or increase the residence time of the particles in the main chamber. As a voltage increase will potentially damage the piezo-electric transducer, a second modular device was fabricated in which the width and length of the main chamber are doubled leading to a factor of 4 increase in the residence time for a given flow rate. Similarly, the transducer area was increased. Concentration measurements were made with fluorescence activated cell sorter (FACS) and, by using it to count

Table 3

Separation results for unmodified modular micro-milled device

\begin{tabular}{|c|c|c|c|c|c|c|c|}
\hline \multicolumn{3}{|c|}{ Flow rate $(\mu \mathrm{l} / \mathrm{min})$} & \multirow{2}{*}{$\begin{array}{l}\text { Transducer } \\
\text { voltage } \\
\left(V_{\text {peak-peak }}\right)\end{array}$} & \multirow{2}{*}{$\begin{array}{l}\text { Particle } \\
\text { diameter } \\
(\mu \mathrm{m})\end{array}$} & \multicolumn{2}{|c|}{$\begin{array}{l}\text { Percentage of } \\
\text { inlet particles }\end{array}$} & \multirow[t]{2}{*}{$\begin{array}{l}\text { Outlet } 2 \\
\text { concentration }\end{array}$} \\
\hline $\begin{array}{l}\text { Inlet } \\
(A)\end{array}$ & $\begin{array}{l}\text { Outlet } \\
1\end{array}$ & $\begin{array}{l}\text { Outlet } \\
2(B)\end{array}$ & & & $\begin{array}{l}\text { Outlet } \\
1(C) \\
(\%) \\
\end{array}$ & $\begin{array}{l}\text { Outlet } \\
2(D) \\
(\%)\end{array}$ & \\
\hline \multicolumn{8}{|c|}{ Horizontal - particles moving against gravity } \\
\hline 100 & 50 & 50 & 10 & 9 & 0 & 79 & 2.0 \\
\hline 100 & 50 & 50 & 10 & 1 & 52 & 42 & 0.9 \\
\hline 100 & 71 & 29 & 10 & 9 & 29 & 47 & 2.1 \\
\hline \multicolumn{8}{|c|}{ Inverted - particles moving with gravity } \\
\hline 100 & 71 & 29 & 10 & 9 & 0 & 83 & 3.4 \\
\hline
\end{tabular}

Table 4

Separation results for modular micro-milled device, modified for the separation of $1 \mu \mathrm{m}$ particles

\begin{tabular}{|c|c|c|c|c|c|c|c|}
\hline \multicolumn{3}{|c|}{ Flow rate $(\mu \mathrm{l} / \mathrm{min})$} & \multirow{2}{*}{$\begin{array}{l}\text { Transducer } \\
\text { voltage } \\
\left(\mathrm{V}_{\text {peak-peak }}\right)\end{array}$} & \multirow{2}{*}{$\begin{array}{l}\text { Particle } \\
\text { diameter } \\
(\mu \mathrm{m})\end{array}$} & \multicolumn{2}{|c|}{$\begin{array}{l}\text { Percentage of } \\
\text { inlet particles }\end{array}$} & \multirow[t]{2}{*}{$\begin{array}{l}\text { Outlet } 2 \\
\text { concentration }\end{array}$} \\
\hline $\begin{array}{l}\text { Inlet } \\
(A)\end{array}$ & $\begin{array}{l}\text { Outlet } \\
1\end{array}$ & $\begin{array}{l}\text { Outlet } \\
2(B)\end{array}$ & & & $\begin{array}{l}\text { Outlet } \\
1(C) \\
(\%)\end{array}$ & $\begin{array}{l}\text { Outlet } \\
2(D) \\
(\%)\end{array}$ & \\
\hline \multicolumn{8}{|c|}{ Horizontal - particles moving against gravity } \\
\hline 100 & 50 & 50 & 10 & 1 & 2 & 84 & 2.0 \\
\hline 100 & 71 & 29 & 14 & 1 & 8 & 74 & 3.1 \\
\hline 100 & 78 & 22 & 12 & 1 & 24 & 63 & 3.3 \\
\hline 100 & 88 & 12 & 14 & 1 & 89 & 11 & 0.9 \\
\hline \multicolumn{8}{|c|}{ Inverted - particles moving with gravity } \\
\hline 100 & 78 & 22 & 14 & 1 & 9 & 71 & 4.0 \\
\hline 100 & 88 & 12 & 14 & 1 & 80 & 18 & 1.5 \\
\hline
\end{tabular}

particles over a known time period, a concentration measurement relative to the inlet can be made.

Tests using the modified design demonstrate separation of $1 \mu \mathrm{m}$ particles, with similar operating conditions to those used previously in Table 3. Table 4 shows that good separation can be achieved at lower flow splits. As the flow split becomes more severe, the separation performance reduces until the majority of particles pass through outlet 1 where the acoustic node and particle stream are positioned beneath the point at which the outlet flow splits. Again a small improvement is seen by inverting the device. The penultimate set of data shown (78:22 flow split) corresponds to a 3.2-fold increase in concentration, or 4.0-fold increase accounting for particles lost in the fluid system.

\section{Discussion}

\subsection{Device fabrication}

In this paper, two fabrication methods have been used to build a suitable resonator. The intention of this study was not to provide a direct comparison between the performances of the two devices, but to demonstrate a progression of the concentrator design such that progressively smaller particles could be concentrated. However, experimental work does reveal how the construction of the devices can influence concentration levels and is explained below.

\subsection{Acoustic performance}

The performance of each concentrator device is highly dependent upon the position of the acoustic node relative to the reflector layer and the uniformity of the nodal plane. In the wet-etched device, lateral forces caused striations to form and create an irregularity in the node position. In the modular device striations were not an obvious feature, however on reaching the first outlet slot the particle stream became distorted which limited the separation performance. Acoustic modes associated with the outlet region may be a possible cause, although more work would be required to ascertain the likely cause.

Lateral radiation forces operating against the direction of flow cause particle trapping and can account for the loss of the $9 \mu \mathrm{m}$ particles. Trapping was also apparent in the wet-etched device between the two outlet ducts where, due to the extraction of fluid through outlet 1 , the flow velocity is significantly lower than in the main chamber thus exacerbating trapping.

In general, the wet-etched and unmodified modular devices did not suffer noticeably from frequency drift and exhibited consistent 
performance at the operating frequency chosen. However, the modified modular device used a transducer which was four times the area of the unmodified version and heating of the transducer was apparent causing a shift in resonance of a $\sim 3 \mathrm{kHz}$ frequency and associated impedance variations. To reduce heating and drive voltage, a thick-film printed transducer instead of the bulk PZT material could be considered.

\subsection{Fluidic design}

The fluidic design of these devices also impacts on their measured performance. Sedimentation of particles is a common problem, reducing the concentration seen through outlet 2 , and is aggravated by the low flow rates used. Reducing cross-sectional areas of the fluidic channels may alleviate this, but increased flow resistances make priming and control of the flow experimentally more challenging.

The size of particles relative to the fluidic features may also determine the performance and outlet concentration. For example, for large particles in systems for which the acoustic node is close to the reflector surface $(<R)$, particles can easily become trapped on the surface and in turn trap more particles due to obstruction or inter-particle forces such as acoustic Bjerknes forces [2].

\section{Conclusions}

With the aid of acoustic modelling, it has been demonstrated that particles can be concentrated using a quarter-wavelength ultrasonic resonance up to 4.4-, 6.0- and 3.2-fold for 9, 3 and $1 \mu \mathrm{m}$ diameter particles. The quarter-wavelength mode can be used to concentrate the majority of suspended particles or to remove large particles which experience a high acoustic radiation force from a suspension of smaller particles.

The operation of such a device is limited by the acoustic properties and size of the particles being separated, and the acoustic energy density of the field. Also, the loss of particles in the system, caused by trapping and fluid handling, has a significant effect on the measured performance. However, further work should concentrate on the position and uniformity of the acoustic node as this appears to be the more important factor in forming a particle concentrate in wide channels using the two outlet approach.

\section{Acknowledgements}

The authors gratefully acknowledge the support and funding from the MoD. Many thanks also go to Mr. Graham Broder, University of Southampton, for his assistance with concentration measurements.

\section{References}

[1] Z.A. Gol'dberg, Acoustic radiation pressure, in: L.D. Rozenburg (Ed.), High Intensity Ultrasonic Fields, Plenum Press, New York, 1971, pp. 75-133.

[2] M. Gröschl, Ultrasonic separation of suspended particles - Part I: Fundamentals, Acustica 84 (1998) 432-447.

[3] M. Wiklund, H.M. Hertz, Ultrasonic enhancement of bead-based bioaffinity assays, Lab on a Chip 6 (2006) 1279-1292.

[4] J.J. Hawkes, M.J. Long, W.T. Coakley, M.B. McDonnell, Ultrasonic deposition of cells on a surface, Biosensors and Bioelectronics 19 (2004) 1021-1028.

[5] S.P. Martin, R.J. Townsend, L.A. Kuznetsova, K.A.J. Borthwick, M. Hill, M.B. McDonnell, W.T. Coakley, Spore and micro-particle capture on an immunosensor surface in an ultrasound standing wave system, Biosensors and Bioelectronics 21 (2005) 758-767. 11/15.

[6] M. Hill, Y.J. Shen, J.J. Hawkes, Modelling of layered resonators for ultrasonic separation, Ultrasonics 40 (2002) 385-392.

[7] R.J. Townsend, M. Hill, N.R. Harris, N.M. White, Modelling of particle paths passing through an ultrasonic standing wave, Ultrasonics 42 (2004) 319-324.

[8] M. Hill, The selection of layer thicknesses to control acoustic radiation force profiles in layered resonators, JASA 114 (2003) 2654-2661.

[9] M. Wiklund, S. Nilsson, H.M. Hertz, Ultrasonic trapping in capillaries for traceamount biomedical analysis, Journal of Applied Physics 90 (2001) 421-426.

[10] A. Haake, J. Dual, Contactless micromanipulation of small particles by an ultrasound field excited by a vibrating body, Journal of the Acoustical Society of America 117 (2005) 2752-2760.

[11] J.J. Hawkes, W.T. Coakley, Force field particle filter, combining ultrasound standing waves and laminar flow, Sensors and Actuators B-Chemical 75 (2001) 213-222.

[12] A. Nilsson, F. Petersson, H. Jonsson, T. Laurell, Acoustic control of suspended particles in micro fluidic chips, Lab on a Chip 4 (2004) 131-135.

[13] F. Petersson, A. Nilsson, C. Holm, H. Jonsson, T. Laurell, Separation of lipids from blood utilizing ultrasonic standing waves in microfluidic channels, Analyst 129 (2004) 938-943.

[14] N.R. Harris, M. Hill, S. Beeby, Y. Shen, N.M. White, J.J. Hawkes, W.T. Coakley, A silicon microfluidic ultrasonic separator, Sensors and Actuators B-Chemical 95 (2003) 425-434

[15] N. Harris, M. Hill, Y. Shen, R.J. Townsend, S. Beeby, N. White, A dual frequency, ultrasonic, microengineered particle manipulator, Ultrasonics 42 (2004) 139144.

[16] R.J. Townsend, M. Hill, N.R. Harris, N.M. White, Investigation of twodimensional acoustic resonant modes in a particle separator, Ultrasonics 44 (2006) e467-e471.

[17] T. Lilliehorn, U. Simu, M. Nilsson, M. Almqvist, T. Stepinski, T. Laurell, J. Nilsson, S. Johansson, Trapping of microparticles in the near field of an ultrasonic transducer, Ultrasonics 43 (2005) 293-303. 